\title{
Grouping by plasmid profiles of atypical Aeromonas salmonicida isolated from fish, with special reference to salmonid fish
}

\author{
Henning Sørum ${ }^{1}$, Gudmund Holstad ${ }^{2, *}$, Tor Lunder ${ }^{2}$, Tore Håstein ${ }^{2}$ \\ ${ }^{1}$ Norwegian School of Veterinary Science, Post Box 8146 Dep., 0033 Oslo, Norway \\ ${ }^{2}$ National Veterinary Institute, Post Box 8156 Dep., 0033 Oslo, Norway
}

\begin{abstract}
Plasmid profile analyses were performed for 113 strains of atypical Aeromonas salmonicida and the reference strain $A$. salmonicida subsp. salmonicida ATCC 14174 . The atypical $A$. salmonicida strains comprised 98 strains obtained from fish originating from 54 farms and 2 lakes in Norway, 10 strains from Canada (2), Denmark (2), Finland (1), Iceland (1) and Sweden (4), the reference strains NCMB 1109 and ATCC 15711 (Haemophilus piscium) of A. salmonicida subsp. achromogenes, and the type cultures A. salmonicida subsp. achromogenes NCMB 1110, A. salmonicida subsp. masoucida ATCC 27013 and A. salmonicida subsp. smithia CCM 4103. A total of 95 strains of atypical $A$. salmonicida were separated into 7 groups (I to VII) based on the plasmid profiles. Eighteen strains of atypical $A$. salmonicida had no common plasmid profile. The type strain NCMB 1110 and the reference strain NCMB 1109 were included in group IV, and the type strain ATCC 27013 in group $V$, but the other reference and type strains had plasmid profiles different from all the other strains. An epidemiological link was documented between strains collected from different farms/localities in each of groups I, III, V and VII. Physiological and biochemical characterizations were performed for 93 of the strains to investigate phenotypic differences between the plasmid groups. Group VII strains and 3 strains with no common plasmid profile differed from the other groups in being catalase-negative. Differences in phenotypic characteristics were shown between the plasmid groups. However, significant variations in reactions for several phenotypic characteristics also occurred within each of the groups I to VII. The present study indicates that plasmid profiling may give useful epidemiological information during outbreaks of atypical A. salmonicida infections in fish. Additional comprehensive phenotypic characterisation is of limited value since the phenotypic characteristics in each plasmid group are not uniform.
\end{abstract}

KEY WORDS: Atypical Aeromonas salmonicida $\cdot$ Fish $\cdot$ Plasmid profiles

\section{INTRODUCTION}

Aeromonas salmonicida comprises facultative anaerobic, Gram-negative, non-encapsulated, non-motile coccobacilli which produce catalase and oxidase, and grow optimally at 22 to $25^{\circ} \mathrm{C}$ (Popoff 1984). Four subspecies, salmonicida, achromogenes, masoucida, and smithia, have been described. Among these, A. salmonicida subsp. salmonicida is the most important fish pathogen. A high level of homology between isolates of this subspecies has been shown based on phenotypic characterisation (McCarthy 1975, Austin et al.

•Corresponding author. E-mail: gudmund.holstad@vetinst.no
1989), plasmid profiling (Bast et al. 1988, Belland \& Trust 1989, Sørum et al. 1993) and DNA-DNA hybridisation (Belland \& Trust 1988)

Strains included in the subspecies achromogenes, masoucida, and smithia, as well as strains not included in any of the described subspecies of Aeromonas salmonicida, are referred to as atypical strains (Wicklund \& Dalsgaard 1998). Several studies have reported isolation of atypical $A$. salmonicida from salmonid and non-salmonid fish (Wiklund \& Dalsgaard 1998). The isolates show variable pigment production (Pedersen et al. 1994), slow growth (Pedersen et al. 1994), nutritional fastidiousness (Austin \& Adams 1996) and growth at elevated temperature (Austin 1993). In Norway, atypical $A$. salmonicida has been isolated from 
arctic char Salvelinus alpinus (Wiklund \& Dalsgaard 1998), halibut Hippoglossus hippoglossus (Gudmundsdóttir 1996), minnow Phoxinus phoxinus (Håstein et al. 1978), turbot Scophthalmus maximus (Gravningen \& Dydland 1991) wolffish Anarhicas lupus (Hellberg et al. 1996), wrasse Labrus berggylta (Gravningen et al. 1996), and frequently from farmed Atlantic salmon Salmo salar kept in seawater net cages (Olsen at al. 1989). The pathological findings include ulcers, boils in the flesh and visceral haemorrhage, and infection with atypical $A$. salmonicida is of economic importance to aquaculture world-wide (Austin \& Austin 1993).

Molecular and phenotypic methods have been used to characterise atypical Aeromonas salmonicida. Austin et al. (1998) found that isolates recovered from a wide range of hosts and geographical locations were heterogeneous. In a study by Pedersen et al. (1996) atypical $A$. salmonicida was characterised by ribotyping and plasmid profiling. Ribotypes were found to be more stable properties than plasmid profiles. Hänninen \& Hirvelä-Koski $(1997,1999)$ found ribopattern analysis and pulsed-field gel electrophoresis to be a valid method to study the epidemiology of infections caused by atypical $A$. salmonicida in fish farms. They also found that plasmid profile analysis was a more sensitive method than ribotyping for the differentiation of pigment-producing strains. Based on this observation they suggested that unique plasmids could probably be used as epidemiological markers to follow the transmission of infection from one fish farm to another.

The aim of the present study was to obtain further epidemiological information about atypical Aeromonas salmonicida using (1) strains from fish in Norway, (2) strains from other countries, and (3) reference and type strains of different $A$. salmonicida subspecies. The strains were primarily grouped by plasmid profiling, and secondarily physiological and biochemical examinations were performed to investigate any phenotypic differences between the plasmid groups.

\section{MATERIALS AND METHODS}

Bacterial strains. A total of 113 strains of atypical Aeromonas salmonicida and the reference strain $A$. salmonicida subsp. salmonicida ATCC 14174 were included (see Table 1). The strains were considered to belong to the species $A$. salmonicida based on preliminary physiological and biochemical characteristics. They were non-motile, Gram-negative coccoid rads, which fermented glucose, grew optimally at 22 to $25^{\circ} \mathrm{C}$, produced cytochrome oxidase, and were resistant to 2,4-diamino-6,7-diisopropylpteridin (O/129). The atypical strains were considered to be atypical $A$. salmonicida if saccharose-positive. Ninety-eight strains of atypical A. salmonicida from the strain collection at the Norwegian National Veterinary Institute were included in the study. Following identification, pure cultures of these strains had been grown on $5 \%$ bovine blood agar incubated aerobically at $22^{\circ} \mathrm{C}$ for 2 to $7 \mathrm{~d}$. Subsequently, they had been grown on Brain Heart Infusion Agar slants and stored at $4^{\circ} \mathrm{C}$. Then, all the strains had been stored in Luria broth (LB) (Miller 1972 ) with $15 \%$ glycerol at $-80^{\circ} \mathrm{C}$ until tested. Most of the Norwegian strains had been obtained from diseased salmonid fish originating from 54 fish farms (F1 to F54) and 2 lakes (Fig. 1). All the strains isolated from a farm during a period of 1 yr were considered to belong to the same disease outbreak in the farm. Only one or a few strains were, however, examined from earh farm during a disease outbreak (Table 1) even though the mortality in the farms was high. The filing procedure as regards material sent to the National Veterinary Institute was as follows: The samples were computer-recorded by name and address of farm, fish species, number and type of samples, date of reception of the sample and type of examination. Paper copies of the filing information with specific forms regarding details of examinations carried out were also made. When examinations had been finalised, the diagnosis response letter and the date of response were filed in the examination documents.

Of the Norwegian strains, 84 originated from Atlantic salmon, 2 from ballan wrasse, 3 from char, 3 from wolffish, and 1 each from bleak Alburnus alburnus, crucian carp Carassius carassius, minnow, roach Rutilus rutilus, sea trout Salmo trutta, and turbot. The Norwegian strains had been isolated from fish in sea water, brackish water, or fresh water. All the chars were from saline water, and all the Atlantic salmon except those originating from farm F47 (Langstein river) were from saline water. Data indicating epidemiological links between strains, i.e. deliveries of fish from one farm to another, were available in some cases for the Norwegian strains. Ten strains of atypical $A$. salmonicida were kindly provided by other laboratories in Sweden (4), Denmark (2), Canada (2), Iceland (1) and Finland (1). Additionally, the reference strains NCMB 1109 and ATCC 15711 (Haemophilus piscium) of Aeromonas salmonicida subsp. achromogenes, and the 3 type cultures, i.e. A. salmonicida subsp. achromogenes NCMB 1110, A. salmonicida subsp. masoucida ATCC 27013, and A. salmonicida subsp. smithia CCM 4103, were examined.

The bacterial strains were separated into groups based on plasmid profiles (Table 1). Strains with the same plasmid profiles and strains containing large and small plasmids of identical size, were included in the same group. To test the stability of plasmid profiles repeated examinations were carried out 2 to 11 times 


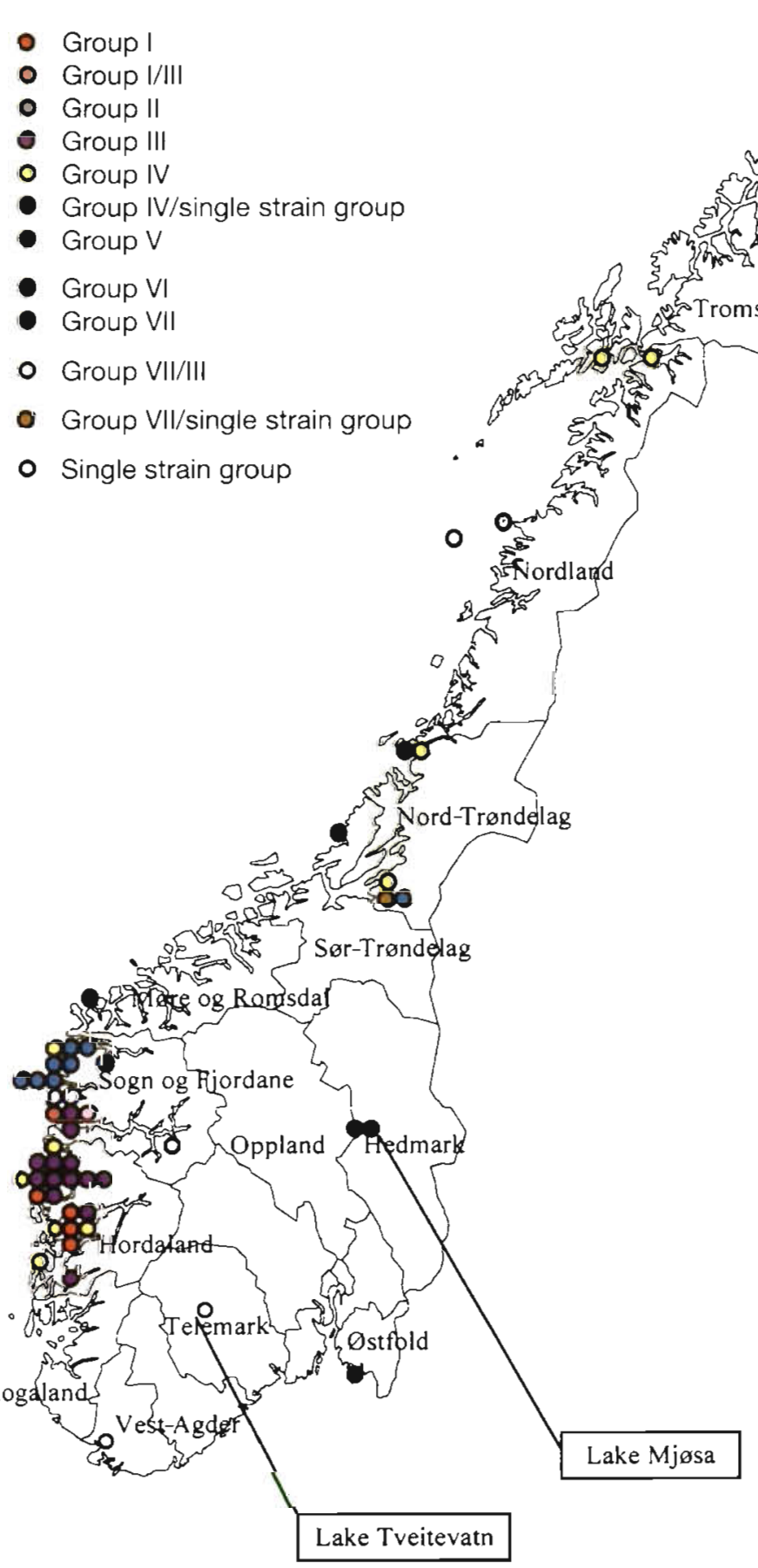

over a period of several months for 84 strains, while for the remaining 30 strains plasmid profiles were examined only once (Table 1). Additionally, physiological and biochemical examinations were carried out with 93 strains to investigate any phenotypic differences between the plasmid groups (see Table 2).

Plasmid isolation. The bacterial strains were incubated in LB at 20 to $22^{\circ} \mathrm{C}$ or at $15^{\circ} \mathrm{C}$ for $2 \mathrm{~d}$ in a roller drum. Plasmid isolation was carried out according to the method of Kado \& Liu (1981). Briefly, agarose gel

electrophoresis was performed in $1 \%(\mathrm{w} / \mathrm{v})$ agarose (SeaKem GTG, FMC Bioproducts, Rockland, ME, USA) gels with Tris-borate-EDTA buffer $(89 \mathrm{mM}$ Tris, $89 \mathrm{mM}$ boric acid, $2.5 \mathrm{mM}$ EDTA [pH 8.0]) at $120 \mathrm{~V}$ (42 $\mathrm{mA})$ for $3 \mathrm{~h}$ at room temperature in a vertical gel apparatus. The agarose gels were stained in ethidium bromide and destained in distilled water. Photographs of the gels were taken under UV-light exposure. The bacterial strains were screened for plasmids over a period of several months. Escherichia coli strain V517 with plasmids of 8 different sizes $(35.8,4.8,3.7,3.4,2.6$, 2.0, 1.8 and 1.4 MDa) (Macrina et al. 1978) and E. coli strain $\mathrm{K} 12$ containing the plasmids pDK9 (140 MDa) (anonymous), R1 (62 MDa) (Bukhari et al. 1977), RP4 (34 MDa) (Bukhari et al. 1977) and Sa (23 MDa) (Bukhari et al. 1977) were used as standards.

Physiological and biochemical characterisation. Physiological and biochemical characteristics examined are presented in Table 2. Cell material for the assays was taken from fresh cultures grown on blood agar plates. Incubations were performed aerobically at $22^{\circ} \mathrm{C}$ and tests were read after $2,5,7$ and $14 \mathrm{~d}$.

Diffusible pigments were observed on Trypticase Yeast extract Agar (TYA), pH 7.0, containing tryptone (Difco) $10 \mathrm{~g}$, yeast extract (Difco) $5 \mathrm{~g}$, and Sobigel-agar (Sobigel, Hendaye Cedex, France) $20 \mathrm{~g}$ in $1 \mathrm{l}$ aqua dest. Autoagglutination was determined using saline cell suspensions on a glass slide. Nitrate reduction was observed on nitrate medium containing tryptone (Difco) $1 \mathrm{~g}$, and nitrite-free $\mathrm{KNO}_{3} 0,2 \mathrm{~g}$ in $1 \mathrm{l}$ aqua dest. The test was read after adding 5 drops of each of reagents $A, 0.5 \%$ alpha-naphthylamine in $5 \mathrm{~N}$ acetic acid, and $\mathrm{B}, 0.8 \%$ sulphanilic acid in $5 \mathrm{~N}$ acetic acid. To determine the production of catalase, a loopful of bacterial growth was taken from the top of the colonies, avoiding the blood agar medium. The bacterial cells were placed on a clean microscopic glass slide and a drop of $3 \%$ hydrogen peroxide was added. Indol production was observed in indol medium, $\mathrm{pH} 7.4$, containing tryptone (Difco) $5 \mathrm{~g}$, and $\mathrm{Na}_{2} \mathrm{HPO}_{4} \cdot 2 \mathrm{H}_{2} \mathrm{O} 2 \mathrm{~g}$ in $1 \mathrm{l}$ aqua dest. The test was read after adding $0.5 \mathrm{ml} \mathrm{Ko-}$ vacs reagent ( $\mathrm{p}$-dimethyl-aminobenzaldehyde [Sigma] $10 \mathrm{~g}$, amyl alcohol [Merck] $150 \mathrm{ml}, \mathrm{HCl}$ [37\%] $50 \mathrm{ml}$ ). 
Table 1. Grouping according to plasmid profiles for 113 strains of atypical Aeromonas salmonicida and the type strain ATCC 14174 of $A$. salmonicida subsp. salmonicida. f: farmed fish; w: wild fish. N: no. of repeated plasmid profiles

\begin{tabular}{|c|c|c|c|c|c|}
\hline $\begin{array}{l}\text { Plasmid group } \\
\text { Collection/strai }\end{array}$ & $\begin{array}{l}\text { Source/farm/county/country of origin } \\
\text { no. }\end{array}$ & $\mathrm{f} / \mathrm{w}^{\mathrm{b}}$ & \multicolumn{2}{|l|}{$\begin{array}{l}\text { Plasmid profiles } \\
\text { (MDa) }\end{array}$} & N \\
\hline \multicolumn{6}{|l|}{ Group I } \\
\hline $87 / 2013$ & Atlantic salmon/F1/Hordaland/Norway & $\mathrm{f}$ & $83,5.1,3.6,3.2$ & & 7 \\
\hline $87 / 2303$ & Atlantic salmon/F1/Hordaland/Norway & $f$ & $83,5.1,3.6,3.2$ & & 4 \\
\hline $89 / 2400$ & Atlantic salmon/F1/Hordaland/Norway & $\mathrm{f}$ & $83,5.1,3.6,3.2$ & & 5 \\
\hline $89 / 2547$ & Atlantic salmon/F2/Hordaland/Norway & $\mathrm{f}$ & $83,5.1,3.6,3.2$ & & 1 \\
\hline $89 / 2725$ & Atlantic salmon/F2/Hordaland/Norway & $\mathrm{f}$ & $83,5.1,3.6,3.2$ & & 3 \\
\hline $89 / 2645$ & Atlantic salmon/F3/Hordaland/Norway & $\mathrm{f}$ & $83,5.1,3.6,3.2$ & & 4 \\
\hline $90 / 115 \mathrm{~F} 1$ & Wrasse/F4/Hordaland/Norway & w & $83,5.1,3.6,3.2$ & & 3 \\
\hline $90 / 2132$ & Atlantic salmon/F5/Sogn and Fjordane/Norway & f & $83,5.1,3.6,3.2$ & & 5 \\
\hline $87 / 2764$ & Atlantic salmon/F6/Troms/Norway & $\mathrm{f}$ & $83,5.1,3.6,3.2$ & & 4 \\
\hline $90 / 115 \mathrm{~F} 2$ & Wrasse/F4/Hordaland/Norway & $w$ & $5.1,3.6,3.2$ & & 3 \\
\hline \multicolumn{6}{|l|}{ Group II _ } \\
\hline $90 / 1711$ & Atlantic salmon/F7/Nordland/Norway & $\mathrm{f}$ & $83,3.9,3.2$ & & 3 \\
\hline $90 / 2029$ & Atlantic salmon/Fr/Nordland/Norway & f & $83,3.9,3.2$ & & 2 \\
\hline \multicolumn{6}{|l|}{ Group III } \\
\hline $89 / 2268$ F605 & Atlantic salmon/F8/Sogn and Fjordane/Norway & f & $73,25,22,16,4.4$ & & 5 \\
\hline $89 / 2268$ F607 & Atlantic salmon/F8/Sogn and Fjordane/Norway & $\mathrm{f}$ & $73,25,22,16,4.4$ & & 2 \\
\hline $89 / 2403684-1$ & Atlantic salmon/F9/Sogn and Fjordane/Norway & $\mathrm{f}$ & $73,25,22,16,4.4$ & & 7 \\
\hline $89 / 2403684-2$ & Atlantic salmon/F9/Sogn and Fjordane/Norway & $\mathrm{f}$ & $73,25,22,16,4.4$ & & 2 \\
\hline $89 / 2724$ & Atlantic salmon/F9/Sogn and Fjordane/Norway & f & $73,25,22,16,4.4$ & & 3 \\
\hline $89 / 3386$ & Atlantic salmon/F10/Sogn and Fjordane/Norway & f & $73,25,22,16,4.4$ & & 4 \\
\hline $89 / 3895$ & Atlantic salmon/F5/Sogn and Fjordane/Norway & f & $73,25,22,16,4.4$ & & 2 \\
\hline $89 / 2402$ & Atlantic salmon/F11/Hordaland/Norway & f & $73,25,22,16,4.4$ & & 11 \\
\hline $89 / 3038$ & Atlantic salmon/F11/Hordaland/Norway & f & $73,25,22,16,4.4$ & & 1 \\
\hline $89 / 3037$ & Atlantic salmon/F12/Hordaland/Norway & $\mathrm{f}$ & $73,25,22,16,4.4$ & & 2 \\
\hline $89 / 3135$ & Atlantic salmon/F13/Hordaland/Norway & $\mathrm{f}$ & $73,25,22,16,4.4$ & & 1 \\
\hline $89 / 3196$ & Atlantic salmon/F14/Hordaland/Norway & f & $73,25,22,16,4.4$ & & 2 \\
\hline $89 / 3384$ & Atlantic salmon/F15/Hordaland/Norway & $\mathrm{f}$ & $73,25,22,16,4.4$ & & 6 \\
\hline $89 / 3447$ & Atlantic salmon/F16/Hordaland/Norway & $\mathrm{f}$ & $73,25,22,16,4.4$ & & 3 \\
\hline $89 / 4057$ & Atlantic salmon/F16/Hordaland/Norway & f & $73,25,22,16,4.4$ & & 2 \\
\hline $89 / 3449$ & Atlantic salmon/F17/Hordaland/Norway & $\mathrm{f}$ & $73,25,22,16,4.4$ & & 3 \\
\hline $89 / 3703$ & Atlantic salmon/F18/Hordaland/Norway & $\mathrm{f}$ & $73,25,22,16,4.4$ & & 3 \\
\hline $89 / 4310$ & Atlantic salmon/F19/Hordaland/Norway & f & $73,25,22,16,4.4$ & & 2 \\
\hline $89 / 4652$ & Atlantic salmon/F20/Hordaland/Norway & f & $73,25,22, \quad 4.4$ & & 1 \\
\hline $89 / 3195$ & Atlantic salmon/F13/Hordaland/Norway & f & $73,25, \quad 16,4.4$ & & 2 \\
\hline $89 / 2723$ & Atlantic salmon/F14/Hordaland/Norway & $\mathrm{f}$ & $73, \quad 22,16,4.4$ & & 2 \\
\hline $89 / 3253$ & Char/F21/Hordaland/Norway & $w$ & $22,16,4.4$ & & 2 \\
\hline $89 / 3254$ & Atlantic salmon/F20/Hordaland/Norway & f & $22,16,4.4$ & & 3 \\
\hline $89 / 2797$ & Atlantic salmon/F22/Sogn and Fjordane/Norway & f & $22, \quad 4.4$ & & 3 \\
\hline $89 / 778$ & Atlantic salmon/F23/Troms/Norway & f & 73 & & 5 \\
\hline $90 / 3106$ & Atlantic salmon/F23/Troms/Norway & $\mathrm{f}$ & 73 & & 1 \\
\hline $89 / 3513$ & Char/F24/Troms/Norway & $w$ & 73 & & 2 \\
\hline $89 / 3655$ & Atlantic salmon/F25/Nord-Trøndelag/Norway & f & 73, & & 2 \\
\hline Group IV & & & & & \\
\hline $\mathrm{NCMB} 1110^{\mathrm{C}}$ & Brown trout & & $61,30,21,11$ & $3.7,3.2,3$ & 2 \\
\hline & & & $61,30,21, \quad 8$ & $3.7,3.2,3$ & 3 \\
\hline $87 / 2778$ & Atlantic salmon/F26/Nordland/Norway & f & $61,30,21,11$ & $3.2,3$ & 1 \\
\hline & & & $61,30,21, \quad 8$ & $3.2,3$ & 2 \\
\hline & & & 61,30 & $3.2,3$ & 7 \\
\hline & & & 61,30 & $3.2,3$ & 2 \\
\hline P85/3280 & Iceland & & $61,30,21$ & $3.2,3$ & 1 \\
\hline & & & $61, \quad 21$, & $3.2,3$ & 1 \\
\hline FT 1842 & Atlantic salmon/F27/Nord-Trøndelag/Norway & $\mathrm{f}$ & $61,30,21$ & $3.2,3$ & 3 \\
\hline FT 1337 & Atlantic salmon/F28/Hordaland/Norway & $\mathrm{f}$ & $61,30,21$ & $3.2,3$ & 1 \\
\hline & & & $61,30,21$ & 3.2 & 1 \\
\hline & & & 61,30 & 3 & 1 \\
\hline NCMB $1109^{d}$ & & & 61,30 & $3.2,3$ & 1 \\
\hline & & & 61, & $3.2,3$ & 4 \\
\hline $69 / 235$ & Atlantic salmon/F29/Hordaland/Norway & $\mathrm{f}$ & 61,30 & $3.2,3$ & 1 \\
\hline
\end{tabular}


Table 1 (continued)

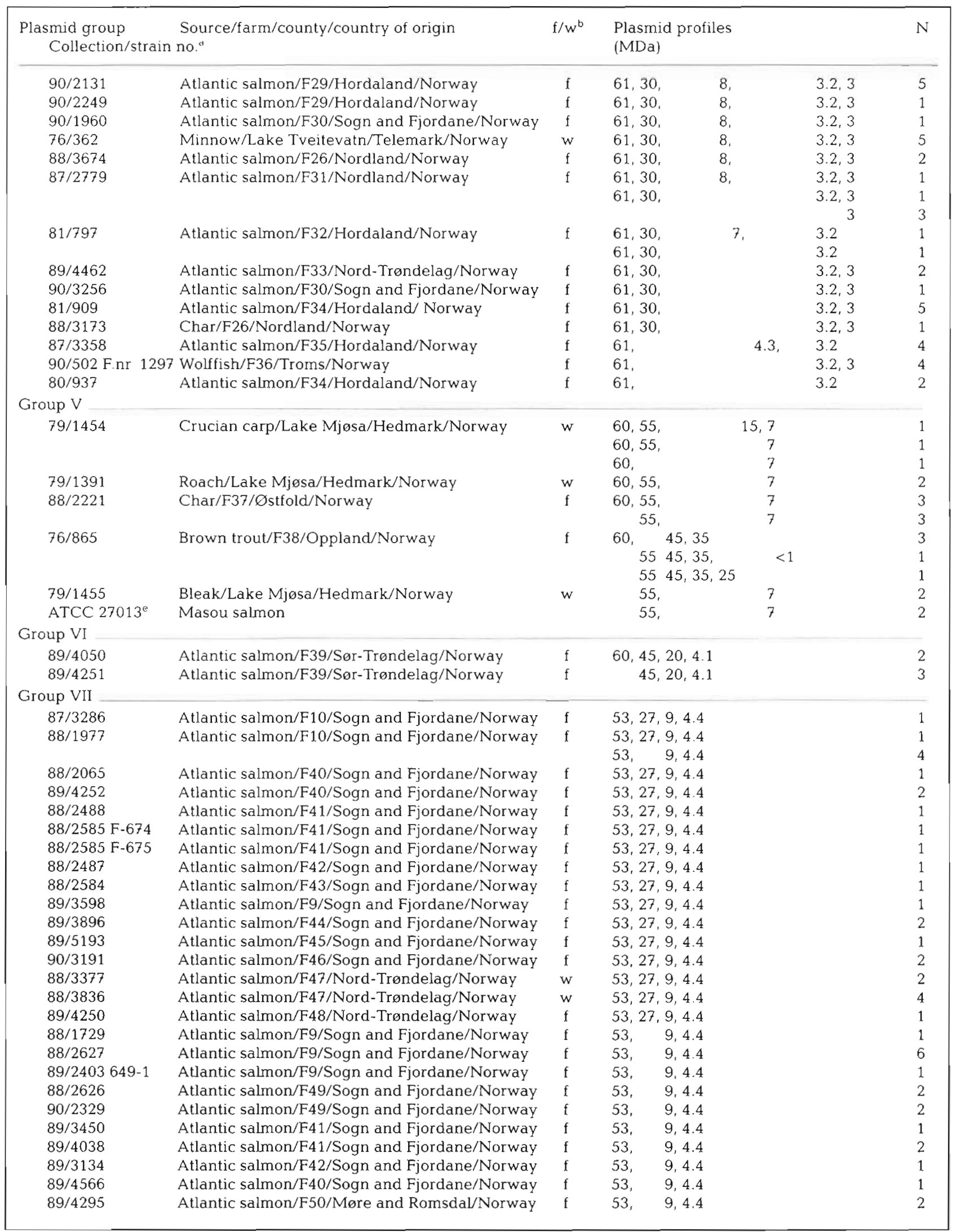


Table 1 (continued)

\begin{tabular}{|c|c|c|c|c|}
\hline $\begin{array}{l}\text { Plasmid group } \\
\text { Collection/strain }\end{array}$ & $\begin{array}{l}\text { Source/farm/county/country of origin } \\
\text { no. }{ }^{a}\end{array}$ & $f / w^{b}$ & $\begin{array}{l}\text { Plasmid profiles } \\
\text { (MDa) }\end{array}$ & $\mathrm{N}$ \\
\hline \multirow{2}{*}{$\begin{array}{l}\text { Single strain groups } \\
\quad 88 / 3835\end{array}$} & & & & \\
\hline & Atlantic salmon/F48/Nord-Trøndelag/Norway & $\mathrm{f}$ & \begin{tabular}{llll}
100, & 15, & 7,6, & \multicolumn{2}{c}{3.6} \\
20, & 12,9, & 4.4 & \\
20, & & 4.4, & 3.3 \\
20, & 12 & & \\
& 12, & 4.4 &
\end{tabular} & $\begin{array}{l}1 \\
1 \\
1 \\
1 \\
5\end{array}$ \\
\hline $87 / 360$ & Sweden & & $85,7,3.2$ & 3 \\
\hline $86 / 369$ & Sweden & & $\begin{array}{ll}83, & 19,14,10,4.5 \\
83, & 19,14,10 \\
65,30, & 14,10,4.5,3.3\end{array}$ & $\begin{array}{l}2 \\
1 \\
1 \\
\end{array}$ \\
\hline 90/502 F.nr.1296 & Wolffish/F36/Troms/Norway & f & $76,15,6.5,1.7$ & 1 \\
\hline $87 / 111$ & Sweden & & 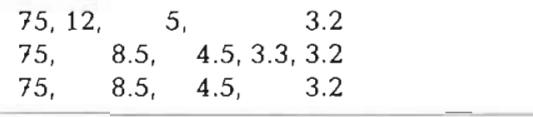 & $\begin{array}{l}1 \\
1 \\
1\end{array}$ \\
\hline Can $870486 \mathrm{~K}$ & Atlantic salmon, Canada & & $74,24,22,21,6.3$ & 2 \\
\hline $86 / 2120$ & Sea trout/F51/Sogn and Fjordane/Norway & w & $\begin{array}{r}20,4.4 \\
61, \quad 20,4.4 \\
55,20,4.4\end{array}$ & $\begin{array}{l}6 \\
2 \\
2\end{array}$ \\
\hline 4425.26 & Finland & & $\begin{array}{ll}72,60,30,19,6 \\
72, & 19,6,3 \\
72, & 19,6 \\
\end{array}$ & $\begin{array}{l}1 \\
4 \\
1 \\
\end{array}$ \\
\hline $86 / 199$ & Sandeel, Denmark & & 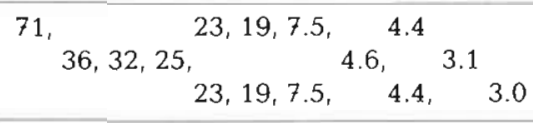 & $\begin{array}{l}3 \\
1 \\
1\end{array}$ \\
\hline 90/502 F.nr.1295 & Wolffish/F36/Troms/Norway & $\mathrm{f}$ & $71,7,2$ & 2 \\
\hline $\mathrm{CCM} 4103^{\mathrm{f}}$ & Roach & & 58,42 & 1 \\
\hline $85 / 2755$ & Rainbow trout, Denmark & & $55,3.5$ & 1 \\
\hline $90 / 2717$ & Turbot/F52/Vest-Agder/Norway & f & $53,44,5,3.2$ & 2 \\
\hline $87 / 448$ & Sweden & & $53,32,18,14,8$ & 3 \\
\hline $89 / 3036$ & Atlantic salmon/F53/Sogn and Fjordane/Norway & $\mathrm{f}$ & $\begin{array}{l}53,19,17,4.4 \\
53,19, \quad 4.4\end{array}$ & $\begin{array}{l}2 \\
1\end{array}$ \\
\hline 90/503 F.nr. 1298 & Atlantic salmon/F54/Nordland/Norway & f & $53,14,6.5,1.7$ & 2 \\
\hline $\operatorname{ATCC} 14174^{\mathrm{g}}$ & Brook trout & & $52,4.2,3.6,3.5,3.4$ & 1 \\
\hline Can $8708924 \mathrm{~K}$ & Atlantic salmon, Canada & & 36,18 & 1 \\
\hline $\operatorname{ATCC} 15711^{b}$ & Brook trout & & $\begin{array}{l}34,29,8 \\
20\end{array}$ & $\begin{array}{l}1 \\
1\end{array}$ \\
\hline $\begin{array}{l}{ }^{a} \text { The figure in front o } \\
\text { bInformation availabl }\end{array}$ & $\begin{array}{l}\text { f the slash mark shows the year of isolation } \\
\text { le only for the Norwegian strains }\end{array}$ & & & \\
\hline $\begin{array}{l}{ }^{\mathrm{C}} \text { A. salmonicida subs } \\
\text { subsp. masowicida tyf } \\
{ }^{\mathrm{h}} \text { A. salmonicida subs }\end{array}$ & $\begin{array}{l}\text { sp. achromogenes type strain, }{ }^{d} \text { A. salmonicida sub } \\
\text { pe strain, }{ }^{\mathrm{f}} \text { A. salmonicida subsp. smithia type strain } \\
\text { p. achromogenes (Haemophilus piscium) reference }\end{array}$ & $\begin{array}{l}\text { sp. ac } \\
\text { gA. sa }\end{array}$ & $\begin{array}{l}\text { omogenes reference strain, }{ }^{e} A \text {. salmoni } \\
\text { nonicida subsp. salmonicida reference str }\end{array}$ & \\
\hline
\end{tabular}

The methyl red and Voges-Proskauer reactions were performed on Clark and Lubs medium containing peptone (Bacto) $5 \mathrm{~g}, \mathrm{~K}_{2} \mathrm{HPO}_{4} 5 \mathrm{~g}$, and D-glucose $5 \mathrm{~g}$ in $1 \mathrm{l}$ aqua dest. The methyl red test was read after adding a few drops of methyl red reagent (methyl red $0.2 \mathrm{~g}$, ethyl alcohol [ $96 \%$ ] $300 \mathrm{ml}$, aqua dest $200 \mathrm{ml}$ ) to $5 \mathrm{ml}$ of
Clark and Lubs medium, while the Voges-Proskauer reaction was read after adding $3 \mathrm{ml} 5 \%$ alpha naphthol in absolute ethyl alcohol and $1 \mathrm{ml} 40 \% \mathrm{KOH}$ to $5 \mathrm{ml}$ of Clark and Lubs medium. For the production of L-arginine decarboxylase, L-lysine decarboxylase and Lornithine decarboxylase, a decarboxylase base solu- 
Table 2. Physiological and biochemical characteristics for strains of atypical Aeromonas salmonicida and the reference strain ATCC 14174 of A. salmonicida subsp. salmonicida. Physiological and biochemical characteristics were not carnied out for strains 90/115 F1, 90/115 F2, 89/2402, 89/4652, 90/3256, 90/3106, P85/3280, FT 1337, 88/3674, 90/3256, 88/3173, 87/3358, 90/502 F.nr. 1297, 90/3191, 90/502 F.nr. 1296, 86/199, 90/502 F.nr. 1295, 85/2755, 90/2717, and ATCC 15711. Previous examinations had shown that all the strains were non-motile, Gram-negative coccoid rods which fermented D-glucose, grew optimally at 22 to $25^{\circ} \mathrm{C}$, produced cytochrome oxidase and were resistant to $\mathrm{O} / 129$ (2,4-diamino-6,7-diisopropylpteridin). All the strains except the type strain ATCC 14174 of $A$. salmonicida subsp. salmonicida fermented saccharose. In the present study all the strains examined of atypical A. salmonicida produced DNase, did not hydrolyse urea, and did not ferment $0.7 \%$ L-arabinose, $0.7 \%$ erythritol, $0.7 \%$ lactose, $0.7 \%$ melibiose, $0.7 \%$ raffinose, $0.7 \%$ L-rhamnose, and $0.7 \%$ salicin + $: 90 \%$ or more are positive; -: $90 \%$ or more are negative; d: $11-89 \%$ are positive. An open space indicates that the examination was not done

\begin{tabular}{|c|c|c|c|c|c|c|c|c|c|c|c|c|c|c|c|c|c|c|c|c|}
\hline 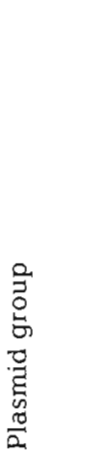 & 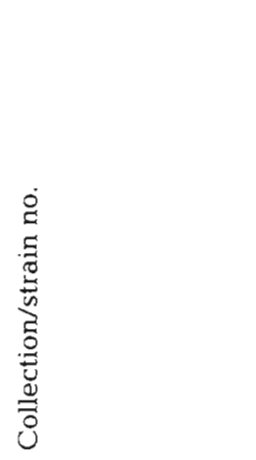 & 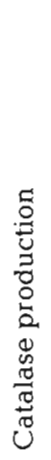 & 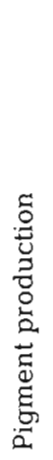 & 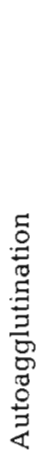 & 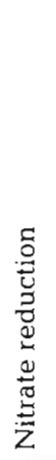 & 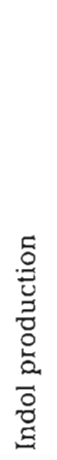 & 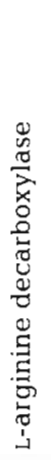 & 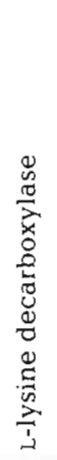 & 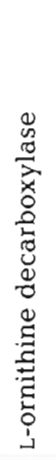 & 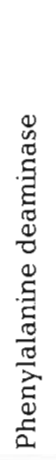 & 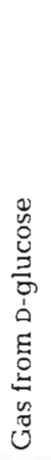 & 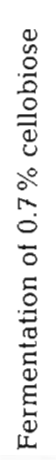 & 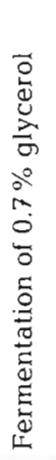 & 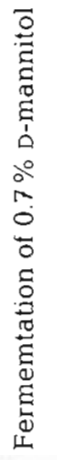 & 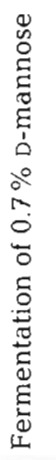 & 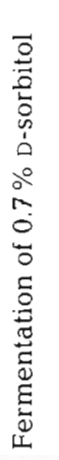 & 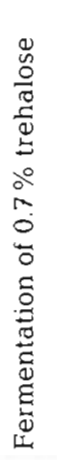 & 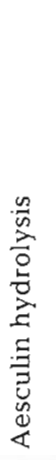 & 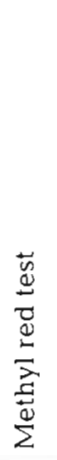 & 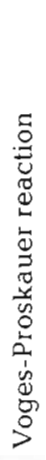 \\
\hline I & & + & + & d & $\mathrm{d}$ & + & - & d & d & d & d & - & $\mathrm{d}$ & + & + & d & + & + & - & + \\
\hline II & & + & - & + & - & - & + & - & - & - & - & - & - & & + & - & $\mathrm{d}$ & d & - & d \\
\hline III & & + & + & + & + & + & - & - & - & - & d & - & + & + & d & - & + & + & d & d \\
\hline IV & & + & d & + & $\mathrm{d}$ & d & d & - & - & $\mathrm{d}$ & - & - & d & + & + & d & + & - & - & d \\
\hline V & & + & - & + & $\mathrm{d}$ & + & & - & - & d & d & $\mathrm{d}$ & $\mathrm{d}$ & + & d & - & d & d & d & d \\
\hline VI & & + & d & d & d & - & - & - & - & d & - & - & - & & + & - & + & - & + & - \\
\hline VII & & - & - & + & - & - & d & - & - & d & - & $\mathrm{d}$ & - & d & d & - & - & d & d & - \\
\hline Single & $88 / 3835$ & - & - & + & - & - & + & - & - & - & - & + & - & + & + & - & - & - & - & - \\
\hline strain & $87 / 360$ & + & + & + & + & - & & - & - & + & - & - & + & + & + & - & + & - & - & - \\
\hline \multirow[t]{11}{*}{ groups } & $86 / 369$ & + & -- & + & + & - & + & - & - & - & - & - & + & + & + & - & + & - & + & - \\
\hline & $87 / 111$ & + & + & + & + & - & & - & - & + & - & - & + & + & + & - & + & - & + & - \\
\hline & Can $870486 \mathrm{~K}$ & + & + & + & + & + & - & - & - & + & - & - & + & + & + & - & + & + & - & + \\
\hline & $86 / 2120$ & + & + & + & + & + & - & - & - & + & - & - & + & + & + & - & + & + & + & + \\
\hline & 4425.26 & + & + & + & + & - & + & - & - & + & - & - & + & + & + & - & + & - & - & - \\
\hline & $\operatorname{CCM} 4103^{\mathrm{a}}$ & + & - & + & & - & - & + & - & & + & & - & & & & - & - & - & \\
\hline & $87 / 448$ & - & - & + & + & - & & - & - & - & - & - & - & + & + & - & - & - & - & - \\
\hline & $89 / 3036$ & + & + & + & + & + & - & - & - & - & - & - & + & & & - & + & - & + & + \\
\hline & $90 / 503$ F.nr. 1298 & - & - & + & - & - & + & - & - & + & & + & - & - & + & - & - & - & + & - \\
\hline & $\operatorname{ATCC} 14174^{\mathrm{b}}$ & + & + & + & + & - & - & + & - & - & + & - & + & + & - & - & + & + & + & - \\
\hline & Can $8708924 \mathrm{~K}$ & + & - & + & - & + & - & - & - & - & - & - & + & + & + & - & + & - & - & - \\
\hline
\end{tabular}

tion was first made using Decarboxylase Base Möller (Difco) $4.2 \mathrm{~g}$ in $400 \mathrm{ml}$ aqua dest. To each of $100 \mathrm{ml}$ slants of this solution L-arginine $\mathrm{HCl} 1 \mathrm{~g}$, L-lysine $\mathrm{HCl}$ $1 \mathrm{~g}$, or L-ornithine $\mathrm{HCl} 1 \mathrm{~g}$ was added. The $\mathrm{pH}$ of the final media was adjusted to 6.0. Phenylalanine deaminase was determined on a medium containing yeast extract (Difco) $3 \mathrm{~g}, \mathrm{~K}_{2} \mathrm{HPO}_{4} 1 \mathrm{~g}, \mathrm{NaCl} 5 \mathrm{~g}$, DL-phenylalanine $2 \mathrm{~g}$, and Agar $12 \mathrm{~g}$ in $1 \mathrm{l}$ aqua dest. The test was read after adding 4 or 5 drops of $10 \%(\mathrm{w} / \mathrm{v}) \mathrm{FeCl}_{3}$ direct to the slant. Production of DNase was determined on Toluidine blue DNA-Agar containing deoxyribonucleic acid $0.3 \mathrm{~g}$. Special Agar Noble $10 \mathrm{~g}, \mathrm{NaCl}$ $10 \mathrm{~g}, 0.01 \mathrm{M} \mathrm{CaCl}_{2} 1 \mathrm{ml}$, and toluidine blue $(6.12 \mathrm{~g}$ in $200 \mathrm{ml}$ aqua dest) $3 \mathrm{ml}$ in $110.05 \mathrm{M}$ Tris- $\mathrm{HCl} \mathrm{pH}$ 9.0.
Production of urease was determined on a urea medium, pH 6.8, containing Bacto Agar (Difco) $7.5 \mathrm{~g}$ and Urea Agar Base solution (Bacto Urea Agar Base [Difco] $290 \mathrm{~g}$ in $1 \mathrm{l}$ aqua dest) $50 \mathrm{ml}$ in $450 \mathrm{ml}$ aqua dest. Fermentation of carbohydrates was determined on a medium containing Hearth Infusion Broth (Difco) $2.5 \mathrm{~g}$, bromthymol blue $(2 \%) 12 \mathrm{ml}$, and $0.7 \mathrm{~g}$ of the actual carbohydrate component in $100 \mathrm{ml}$ aqua dest. Gas production from D-glucose was observed using an inverted Durham tube in the medium. Aesculin hydrolysis was determined on aesculin broth containing peptone (Bacto) $5 \mathrm{~g}$, and aesculin $1 \mathrm{~g}$ in $1 \mathrm{l}$ aqua dest. The test was read by adding a few drops of $1 \% \mathrm{FeCl}_{3}$ to the medium. 


\section{RESULTS}

\section{Plasmid isolation}

Plasmid profiles of the 114 strains are presented in Table 1 A total of 95 strains of atypical Aeromonas salmonicida were separated into 7 groups (I to VII) based on the plasmid profiles. However, 18 strains of atypical $A$. salmonicida and the reference strain $A$. salmonicida subsp. salmonicida ATCC 14174 had no common profile with other strains and constituted 19 different sungle isolate groups. The geographical distribution of the Norwegian fish farms and the 2 lakes containing different plasmid groups is presented in Fig. 1.
All the strains in groups I, II, III and VI that were examned twice or more showed identical plasmid profiles during repeated examinations (Table 1). In group IV, 7 out of 16 strains examined more than once showed different plasmid profiles during repeated examinations. The corresponding figures in the other groups were: group V, 3 out of 6 , group VII, 1 out of 11 ; single strain groups, 8 out of 14 .

Out of 10 strauns in group I, 9 had identical plasmid profiles, and 8 of these strains originated from farmed Atlantic salmon (Table 1). Two strains were detected from 2 different wrasse during a disease outbreak in Atlantic salmon in farm F4, and one of these strains (90/115 F2) lacked a plasmid of $83 \mathrm{MDa}$.
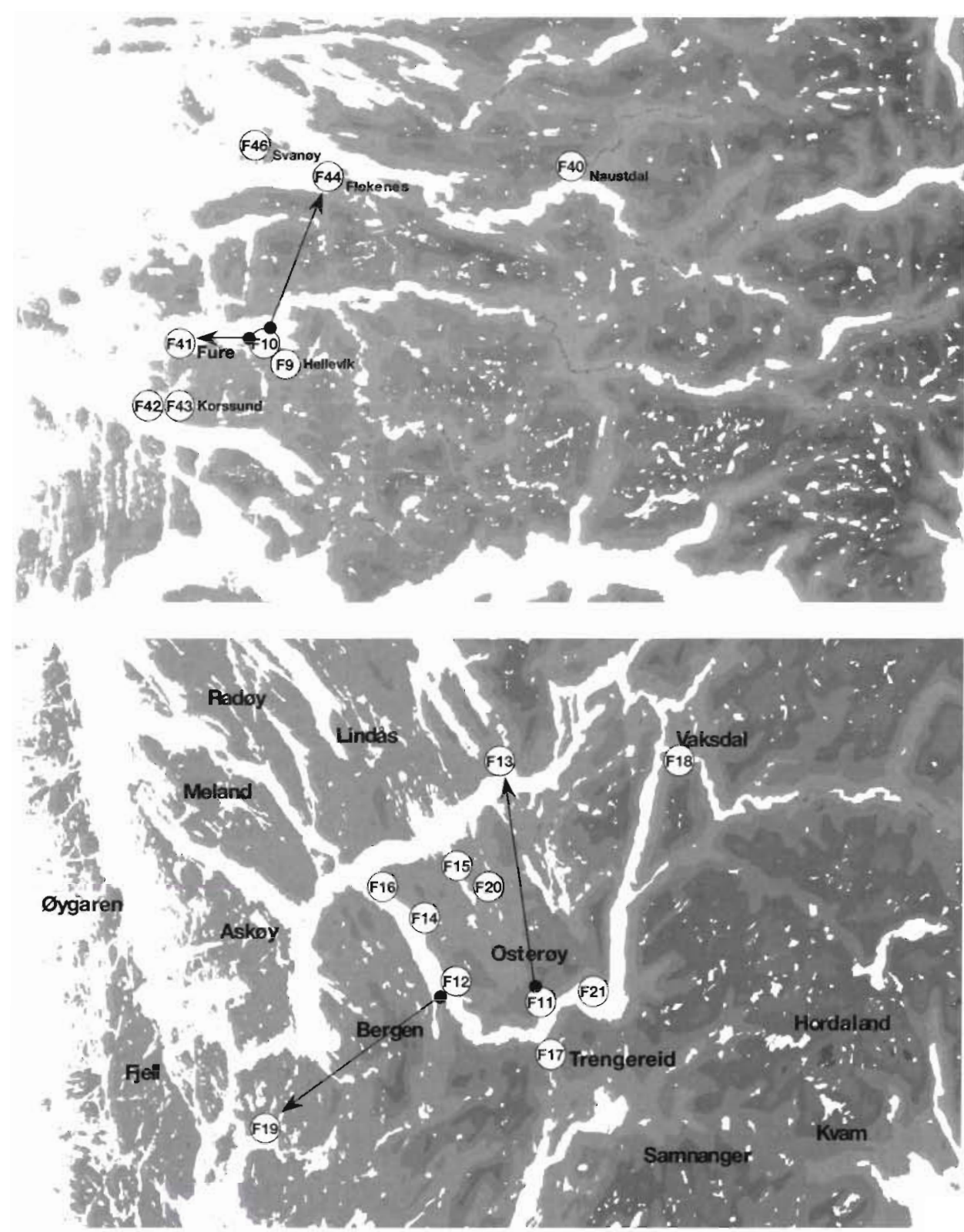

Fig. 2. Locations of 8 fish farms in Sogn and Fjordane county, Norway. Two farms, F9 and F10, contained strains included in both plasmid groups III and VII, while the other farms contained strains only from plasmid group VII. Arrows indicate possible contact between farms
Fig. 3. Locations of all the farms in plasmid group III from Hordaland county, Norway. Arrows indicate possible contacts between farms 
The 2 strains in group II originated from 2 different farmed Atlantic salmon in a single farm (F7) (Table 1). These strains contained 2 plasmids, 83 and $3.2 \mathrm{MDa}$, with the same size as 2 plasmids found in group I. There was also a close link between all the strains in group I and between the strains in groups I and II as farm $F 1$ had delivered smolts to the farms in these 2 groups.

Group III comprised 28 strains, 26 from farmed Atlantic salmon and 2 from char, isolated during a period of 1 yr (Table 1). The occurrence of 3 of the plasmids, 25,22 and $16 \mathrm{MDa}$, varied between some of the strains. It is noteworthy that in farm F5 one isolate (89/3895) was found to belong to group III, while another (90/ 2132) had a plasmid profile in accordance with group I. Epidemiological information indicated that fish from farm F9 in Sogn and Fjordane county had been placed in a net cage close to a net cage containing fish originating from farm F11 in Hordaland county (Figs. 2. \& 3). Further farms, F9 and F10, in Sogn and Fjordane county are situated near to each other and thus a spread of infection through sea water might have been possible. From both these farms there were also several strains included in group VII (Table 1). For the other group III farms in Sogn and Fjordane county no information as regards possible links between strains from different farms was available. There was, however, a direct or indirect link between all the strains from farms in Hordaland county in group III. As can be seen from Fig. 3, 7 of the farms (F11, F12, F14 to F16, F20 and F21) are located around the Osterøy, which is an inland island with low exchange of sea water, while 3 further farms (F13, F17 and F18) are located in the fjords surrounding the island. It could be traced back that infected smolt had been delivered from farm F11 to farm F13 and from farm F12 to farm F19. The proximity of the farms in this region also indicates that a spread of bacterial clones through the sea water might have been possible. There was no information available about any link between the 2 strains from F23 in Troms county and other fish farms in group III. These strains had, however, identical plasmid profiles to strain 89/3513 found in farm F24 in Troms county. The latter strain had been isolated from char, which originated from wild caught char stocked from Lake Takvatn. This finding indicates that a separate clone of this type of atypical Aeromonas salmonicida exists in this area as there was no indication of restocking of fish from Hordaland or Sogn and Fjordane counties into the lake.

Group IV included altogether 21 strains, 18 from Norway, 1 from Iceland, and the type strain NCMB 1110 and the reference strain NCMB 1109 of Aeromonas salmonicida subsp. achromogenes (Table 1). Out of the Norwegian strains, 17 had been isolated from farmed fish and 1 (76/362) from wild fish over a period of $22 \mathrm{yr}$. Altogether 10 different plasmids, of 61 , $30,21,11,8,7,4.3,3.7,3.2$ and $3 \mathrm{MDa}$, were demonstrated in this group. Variation in the occurrence of plasmids was shown both between strains and for repeated examinations of the same strains. The medium sized plasmids were especially unstable. There was no information available about any links between the strains in group IV from the different Norwegian farms. Nor was there data available that indicated any connection between the Norwegian strains and the isolate from Iceland. Our findings show that group IV strains are widely spread in Norway (Fig. 1) and probably internationally.

Group V included the type strain ATCC 27013 of Aeromonas salmonicida subsp. masoucida and 5 Norwegian strains isolated during a period of $13 \mathrm{yr}$ (Table 1). Eight different plasmids, of $60,55,45,35,25$, 15,7 and $<1 \mathrm{MDa}$, were demonstrated in this group, and significant variation in the occurrence of plasmids was shown on repeated examinations for 3 strains. Both strain 79/1455 and strain ATCC 27013 lacked the plasmid of $60 \mathrm{MDa}$. Three Norwegian strains were isolated from 3 different species of wild fishes in Lake Mjøsa (Fig. 4), while 2 Norwegian strains (76/865 and $88 / 2221$ ) were isolated from farmed brown trout and char, respectively. The brown trout was isolated from a hatchery (F38) situated on the river Hunnselva (Fig. 4). This river drains into Lake Mjøsa. Similarly, the char was from a farm (F37) situated near the estuary of the river Glomma (Fig. 4). The river Vorma, a tributary of the Glomma, drains from Lake Mjøsa. Thus, there might be an epidemiological link between all the Norwegian strains in group $V$.

Group VI included only 2 strains from a single farm (F39) (Table 1). Plasmids with the sizes 45 and $60 \mathrm{MDa}$ were also found in strains in group $\mathrm{V}$.

Group VIl contained 26 strains isolated over a period of 4 yr (Table 1). Most of the strains were, however, isolated during 1988 and 1989. Four plasmids, of 53, 27, 9 and 4.4 MDa, were isolated in group VII. The $27 \mathrm{MDa}$ plasmid was present in 16 strains, while the other plasmids were present in all the strains. Twenty-four strains were detected from farmed Atlantic salmon originating from 12 farms in 3 different counties while 2 strains were isolated from wild Atlantic salmon in the Langstein river in Nord Trøndelag county. There was a link between some of the strains in group VII isolated from fish from farms in Sogn and Fjordane county because it could be traced back that infected smolt had been delivered from F10 to F41 and F44 (Fig. 2). For the other farms in group VII no such epidemiological information was available, but some of these farms (F40, F42, F43, and F46) were also located in the same area and spread of bacterial clones through the sea 
water might have been possible. One strain (89/4250) from Nord Trøndelag county was isolated from Atlantic salmon from a farm (F48) located in the same area as the Langstein river. Since one of the strains in Langstein river (88/3377) was isolated from an escaped farm-fish it is uncertain whether the farm or the Langstein river was the primary source of infection

The single isolate groups had no common plasmid profile (Table 1). Seven strains originated from Norwegian farms, and 2 of the strains were isolated at the same time from 2 different wolffish in 1 farm (F36). The other 5 Norwegian strains were isolated from 5 farms in different regions of the country. One of these strains (88/3835) came from a farm (F48) where a group VII strain (89/4250) was also isolated. Strain 89/3036 contained 2 piasnidus, 53 and $4.4 \mathrm{MiDd}$, which were also found in all group VII strains, and strain 89/3036 should therefore in fact have been included in group VII. The strain, however, differed from group VII in many of the phenotypic characteristics, as well as the catalase reaction (Table 2 ). The single isolate groups also contained the 4 Swedish strains, the 2 Danish strains, the 2 Canadian strains, and the strain from Finland. The type strain Aeromonas salmonicida subsp. smithia CCM 4103, and the reference strains A. salmonicida subsp. achromogenes ATCC 15711, and A. salmonicida subsp. salmonicida ATCC 14174 were also included in the groups of single strains.

\section{Physiological and biochemical characterisation}

Results of physiological and biochemical characterisation are presented in Table 2. Variations in reactions for phenotypic characteristics were observed among plasmid groups I to VII and also within each of these groups. Only for the catalase reaction was there either a positive or negative reaction for each of the plasmid groups; the other characteristics showed variations in 1 or more of these groups. All the strains in group VII and 3 single isolate groups -2 Norwegian (90/503 Fnr. 1298 and 88/3835) and 1 Swedish (87/448)-were catalase-negative.

The type strain NCMB 1110 and the reference strain NCMB 1109 of Aeromonas salmonicida subsp. achromogenes differed from the group IV strains in some phenotypic characteristics, but these characteristics also varied among the other strains in group IV. The type strain ATCC 27013 of A. salmonicida subsp. masoucida reduced nitrate, produced phenylalanine deaminase and gas from D-glucose, fermented glycerol and trehalose, hydrolysed aesculin, and showed a positive Voges-Proskauer reaction. All the other isolates in group $\mathrm{V}$ showed a uniform negative reaction for all these characteristics.

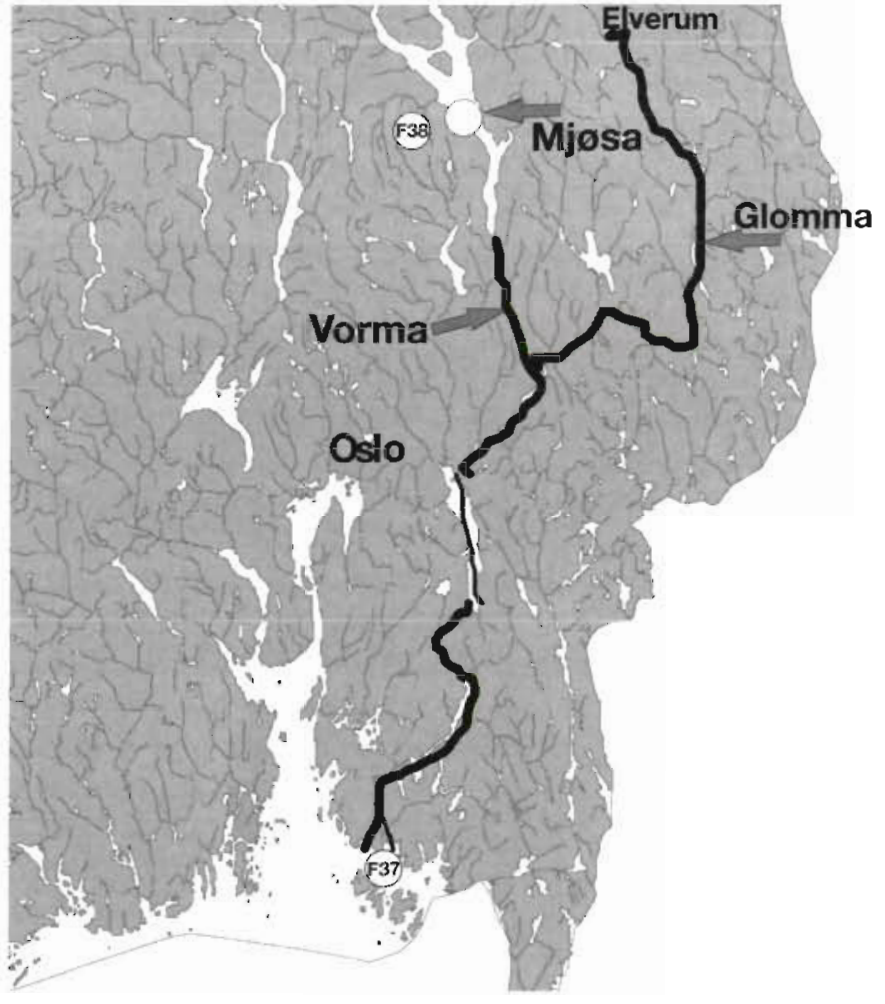

Fig. 4. Locations of farms in plasmid group $V$ and the possible relation between farm F38 strain, Lake Mjøsa strains, and (via the rivers Vorma and Glomma) farm F37 strain

\section{DISCUSSION}

Aeromonas salmonicida subsp. salmonicida is genetically homogenic throughout the world, and plasmid profiling is of little value in identifying the source of infection during outbreaks of furunculosis in salmonids (Sørum et al. 1993). The present study shows, however, that the plasmid profiles vary between isolates of atypical $A$. salmonicida, an observation also shown by Bast et al. (1988), Pedersen et al. (1996), Hänninen \& Hirvelä-Koski (1997), and Austin et al. (1998). The majority of atypical A. salmonicida strains (95) could be divided into 7 different groups (I to VII) based on similarity in plasmid profiles. Physiological and biochemical characteristics varied between the plasmid groups but significant phenotypic variation was also shown for some characteristics within each of these groups. Therefore, phenotypic characterisation is of limited value in differentiating between the plasmid groups. Other authors have also found that atypical A. salmonicida is phenotypically heterogeneous (Austin et al. 1998). A peculiar finding in our study was that none of the strains in group VII produced catalase.

Plasmids belong to the mobile part of the bacterial DNA and often variation is the nature of plasmid DNA 
(Fernandez-Astorga et al. 1992). This variation is also the key to the use of plasmid profiling in epidemiological mapping of infectious diseases. Variation in plasmid content often occurs in strains, but within a clonal group this will occur in such a way that one still may recognise a group connection even if a plasmid is lost or gained. In groups I, III, IV, V, VI and VII some variations in plasmid profiles between different strains were found. These variations were most prominent for medium sized plasmids, indicating that these plasmids are more unstable than the large and small ones. Plasmids containing antimicrobial resistance genes are often mobile, being transferred between bacteria (Kruse \& Sørum 1994). Recombinations where 2 separate plasmids join at homologue sites to make only 1 plasmid have also been demonstrated (Berg et al. 1998). Further investigations have shown that the medium sized plasmids in groups III (25 MDa), IV ( $8 \mathrm{MDa}$ ) and VII (27 MDa) are R-plasmids (data not shown).

Several strains in some of the groups had been isolated over a period of many years, while other strains were isolated at the same time (Table 1). No difference in variation in plasmid profiles between strains isolated several years apart and strains isolated at the same time could be detected. Many strains had also been stored for several years before the plasmid isolations were performed. Sørum et al. (1993) found that the age of the culture did not appear to account for variability in large plasmids in Aeromonas salmonicida subsp. salmonicida. The variation within the plasmid groups may, however, result from the fact that one single plasmid may exist in different conformational variants simultaneously (Sørum et al. 1993).

In this study repeated examinations of the same strains indicated plasmid stability for strains in groups I, II, III and VII, while several strains in groups IV and V and the strains in single strain groups showed different plasmid profiles during repeated examinations. Variation in the plasmid profile was especially prominent in the single strain groups. These findings indicate that repeated examinations of the same strains should be performed if plasmid profiles are used as fingerprints to obtain epidemiological information about outbreaks of atypical Aeromonas salmonicida infection in fish.

Some of the strains examined in the present study have also been examined for plasmids by other authors. Austin et al. (1998) examined strains 87/2303, 88/2221, 88/3377, 89/2403, CCM 4103, 90/2717 and ATCC 14174 and found $4,2,2,4,3,2$ and 3 plasmids in each strain respectively, while the corresponding figures in the present study were $4,3(2), 4,3,2,4$ and 5 . Four of these strains were examined twice or more, while 3 strains were examined only once in our study. Austin et al. (1998) used the kb unit to give the size of the plasmids, and a direct comparison of the size of the plas- mids in the 2 different investigations is therefore difficult. However, converting from $\mathrm{kb}$ to MDa by dividing by a factor of 1.5 , one can see that strain $87 / 2303$ is reported with approximately the same sizes of plasmids. Similarly, strain $88 / 2221$ is reported by Austin et al. (1998) as having 2 plasmids of approximately the same size as 2 of the 3 plasmids in our study. The 2 plasmids in strain 88/3377 reported by Austin et al. (1998) correspond to the largest and the smallest plasmid of the same strain in our study. The $27 \mathrm{MDa}$ plasmid in our investigation may have been lost during storage and handling. In the study by Austin et al. (1998) strain 89/2403 had a completely different plasmid profile, while strain CCM 4103 had 1 plasmid similar to our investigation. Austin et al. (1998) found that strain $90 / 2717$ had 2 plasmids, which probably correspond to the smallest plasmids observed in our study. Pedersen et al. (1996) analysed strain NCMB 1110 and detected 3 plasmids, the corresponding figure in our study being 8 plasmids. Bast et al. (1988) examined NCMB 1110 and found 4 plasmids, of $65,31,3.7$ and 3 MDa. These figures correspond well with the sizes for $4(61,30,3.7$ and $3 \mathrm{MDa})$ out of the 8 plasmids found in this strain in our study (Table 1). Bast et al. (1988) also examined strain ATCC 14174 and found 5 plasmids, of 50 to $56,4.2,3.6,3.5$ and $3.3 \mathrm{MDa}$. These figures also correspond well with the findings of our study (Table 1). The discrepancies in the number of plasmids for the same strains found in different studies is not unexpected in view of the variations found during repeated examinations in our own study. However, these discrepancies may also be due to methodological differences between different laboratories. Thus, results from plasmid isolations carried out in different studies should be compared with caution.

An epidemiological link could be demonstrated between strains in several groups, supporting the hypothesis that each group might represent the same clone of the atypical Aeromonas salmonicida. Our study indicates that a bacterial clone can be spread by transfer of fish between different farms, and probably also through sea and fresh water. Another explanation can, however, be that the same bacterial clones of atypical $A$. salmonicida inhabit different locations in sea or fresh water, and might under certain management conditions cause disease outbreaks.

All the strains from outside Norway, except the isolate from Iceland (group IV), were single isolate groups, suggesting that most of these strains represent clones different from the Norwegian ones. Group IV strains were found in different regions of Norway, and there was no information available that indicated any epidemiological link between strains in this group. Group IV strains had, however, the same plasmid profiles as the Aeromonas salmonicida subsp. achromo- 
genes type strain. This observation implies that the group IV strains could be included in the subspecies achromogenes. This subspecies may, like A. salmonicida subsp. salmonicida, be widespread throughout the world. A. salmonicida subsp. masoucida ATCC 27013 was included in group $V$. However, since this type strain differed from the group $V$ strains in 7 phenotypic characteristics, the group $V$ strains should not be included in the subspecies masoucida.

There was no relation between fish species and plasmid groups since different plasmid groups were detected in the same fish species in our study. Wild fish with contacts with farmed fish may be infected with the same clones of atypical Aeromonas salmonicida and vice versa.

Since strains in groups I and It had similar plasmid profiles and an epidemiological connection could be demonstrated between farms in these groups, a fusion between the 2 groups might be reasonable. The phenotypic examinations, however, showed that the group I strains produced pigment, while the group II strains did not. Plasmids with the same size were also isolated in groups V and VI. More epidemiological information about the strains should, however, be collected before these groups are fused.

The present study suggests that each plasmid group represents a single clone. Austin et al. (1998) examined 52 isolates of atypical Aeromonas salmonicida from a wide range of hosts and geographical locations. They found that most of the isolates had different plasmid profiles and thus probably represented different clones. In the present study we found that strains with different plasmid profiles could be isolated during the same disease outbreak in a farm (F9). In an investigation by Pedersen et al. (1996) it was also concluded that plasmid profiles varied between isolates from the same outbreak. Therefore several isolates from different fish should be examined during disease outbreaks with atypical $A$. salmonicida in a farm.

The atypical strains show great variation in plasmid profiles, in contrast to typical Aeromonas salmonicida strains, which show almost identical profiles all around the world. Several different atypical strains tend to cause various outbreaks. Our study indicates that comprehensive analysis of plasmid profiles can give useful epidemiological information in evaluation of outbreaks of disease caused by atypical $A$. salmonicida.

Acknowledgements. We thank Dr A. B. Olsen, National Veterinary Institute Bergen, Norway, Drs O. Ljungberg and U. P. Wichardt, Sweden, Dr I Dalsgaard, Denmark, the late P. Vestergaard Jørgensen, Denmark, Dr R. Stevenson, Canada, Dr S. Helgason, Iceland, and Dr P. Koski, Finland, for their kindness in making bacterial isolates available to us. We also acknowledge the technical assistance of Cathrine Fjellvang.

\section{LITERATURE CITED}

Austin B (1993) Recovery of 'atypical' isolates of Aeromonas salmonicida, which grow at $37^{\circ} \mathrm{C}$, from ulcerated nonsalmonids in England. J Fish Dis 16:165-168

Austin B, Adams C (1996) Fish pathogens. In: Austin B Altwegg $\mathrm{M}$, Gosling $\mathrm{PJ}$, Joseph $\mathrm{S}$ (eds) The genus Aeromonas. John Wiley \& Sons, Chichester, p 197-243

Austin B, Austin DA (1993) Bacterial fish pathogen diseases of farmed and wild fish, 2nd edn. Ellis Horwood, Chichester

Austin B, Austin DA, Dalsgaard I, Gudmundsdóttir BK, Høie S, Thornton JM, Larsen JL, O'Hici B, Powell R (1998) Characterization of atypical Aeromonas salmonicida by different methods. System Appl Microbiol 21:50-64

Austin DA, McIntosh D. Austin B (1989) Taxonomy of fish associated Aeromonas spp., with the description of Aeromonas salmonicida subsp. smithia subsp. nov. Syst Appl Microbiol 11:277-290

Bast L, Daly JG, DeGrandis SA, Stevenson RMW (1988) Evaluation of profiles of Aeromonas salmonicida as epidemiological markers of furunculosis infections in fish. J Fish Dis 11:133-145

Belland RJ, Trust TJ (1988) DNA:DNA reassociation analysis of Aeromonas salmonicida. J Gen Microbiol 134:307-315

Belland RJ, Trust TJ (1989) Aeromonas salmonicida plasmids: plasmid-directed synthesis of proteins in vitro and in Escherichia coli minicells. J Gen Microbiol 135:513-524

Berg T, Firth N, Apisiridej S, Hettiaratchi A, Leelaporn, Skurray RA (1998) Complete nucleotide sequence of pSK41: evolution of staphylococcal conjugative multiresistance plasmids. J Bacteriol 180:4350-4359

Bukhari AI, Shapiro JA, Adhya SL (1977) DNA insertion elements, plasmids, and episomes. Cold Spring Harbor Laboratory, Cold Spring Harbor, NY

Fernandez-Astorga A, Muela A, Cisterna R, Iriberri J, Barcina I (1992) Biotic and abiotic factors affecting plasmid transfer in Escherichia coli strains. Appl Environ Microbiol 58: 392-398

Gravningen K, Dydland M (1991) Diseases in turbot (Scophthalmus maximus L). Nor Veterinærtidsskr 103:923-926

Gravningen K, Kvenseth PG, Hovlid RO (1996) Virulence of Vibrio anguillarum serotypes $\mathrm{O} 1$ and $\mathrm{O} 2$, Aeromonas salmonicida subsp. salmonicida and atypical Aeromonas salmonicida to goldsinny wrasse. In: Sayer MDJ, Treasure JW, Costello MJ (eds) Wrasse: biology and use in aquaculture. Fishing News Books, Farnham, p 247-250

Gudmundsdóttir BK (1996) Comparison of extracellular proteases produced by Aeromonas salmonicida strains, isolated from various fish species. J Appl Bacteriol 80: $105-113$

Hänninen ML, Hirvelä-Koski V (1997) Molecular and phenotypic methods for the characterization of atypical Aeromonas salmonicida. Vet Microbiol 56:147-158

Hänninen ML, Hirvelä-Koski V (1999) Genetic diversity of atypical Aeromonas salmonicida studied by pulsed-field gel electrophoresis. Epidemiol Infect 123:299-307

Håstein T, Saltveit SJ, Roberts RJ (1978) Mass mortality among minnows Phoxinus phoxinus (L.) in Lake Tveitevatn, Norway, due to an aberrant strain of Aeromonas salmonicida. J Fish Dis 1:241-249

Hellberg H, Moksness E, Høie S (1996) Infection with atypical Aeromonas salmonicida in farmed common wolffish, Anarhichas lupus L. J Fish Dis 19:329-332

Kado CI, Liu ST (1981) Rapid procedure for detection and isolation of large and small plasmids. J Bacteriol 145: $1365-1373$ 
Kruse H, Sørum H (1994) Transfer of multiple drug resistance plasmids between bacteria of diverse onigins in natural microenvironments. Appl Environ Microbiol 60: $4015-4021$

Macrina FL, Kopecko DJ, Jones KR, Ayers DJ, McCowen SM (1978) A multiple plasmid-containing Escherichia coli strain: convenient source of size reference plasmid molecules. Plasmid 1:417-420

McCarthy DH (1975) Fish furunculosis caused by Aeromonas salmonicida var achromogenes. J Wildl Dis 11:489-493

Miller JH (1972) Experiments in molecular genetics. Cold Spring Harbor Laboratory, Cold Spring Harbor, NY

Olsen $A B$, Lunder T, Reitan LJ (1989) Infection with a particular strain of atypical Aeromonas salmonicida in Norway. Abstracts book, European Association of Fish Pathologists 4 th International Conference 'Diseases of fish and shellfish', Santiago de Compostela, Spain, 24-28 September 1989, p 199

Editorial responsibility: David Bruno,

Aberdeen, Scotland, UK
Pedersen K, Kofod H, Dalsgaard I, Larsen JL (1994) Isolation of oxidase-negative Aeromonas salmonicida from diseased turbot Scophthalmus maximus. Dis Aquat Org 18 $149-154$

Pedersen K, Dalsgaard I, Larsen JL (1996) Characterization of atypical Aeromonas salmonicida isolates by ribotyping and plasmid profiling. J Appl Bacteriol 80:37-44

Popoff M (1984) Genus III. Aeromonas Kluyver and Van Niel 1936, 398 AL In: Krieg NR, Holt JG (eds) Bergey's manual of systematic bacteriology, Vol 1. Williams \& Wilkins, Baltimore, p 545-548

Sørum $\mathrm{H}$, Kvello JH, Håstein $\mathrm{T}$ (1993) Occurrence and stability of plasmids in Aeromonas salmonicida ss salmonicida isolated from salmonids with furunculosis. Dis Aquat Org 16:199-206

Wiklund T, Dalsgaard I (1998) Occurrence and significance of atypical Aeromonas salmonicida in non-salmonid and salmonid fish species: a review. Dis Aquat Org 32:49-69

Submitted: January 5, 1999; Accepted: March 14, 2000 Proofs received from author(s): June 7, 2000 